AcCepted for publication in Astrophysical Journal, July 23, 2015

Preprint typeset using $\mathrm{A}_{\mathrm{A}} \mathrm{EX}$ style emulateapj v. 5/2/11

\title{
SHOCK WAVE STRUCTURE IN ASTROPHYSICAL FLOWS WITH AN ACCOUNT OF PHOTON TRANSFER
}

\author{
Alexey Tolstov ${ }^{1,2, *}$, Sergei Blinnikov ${ }^{1,2,3}$, Shigehiro Nagataki ${ }^{4}$, Ken'ichi Nomoto $^{1,5}$ \\ ${ }^{1}$ Kavli Institute for the Physics and Mathematics of the Universe (WPI), The University of Tokyo Institutes for Advanced Study, The \\ University of Tokyo, 5-1-5 Kashiwanoha, Kashiwa, Chiba 277-8583, Japan \\ 2 ITEP, 117218 Moscow, Russia \\ 3 VNIIA, 127055 Moscow, Russia and \\ 4 Astrophysical Big Bang Laboratory, RIKEN, Saitama 351-0198, Japan \\ Accepted for publication in Astrophysical Journal, July 23, 2015
}

\begin{abstract}
For an accurate treatment of the shock wave propagation in high-energy astrophysical phenomena, such as supernova shock breakouts, gamma-ray bursts and accretion disks, knowledge of radiative transfer plays a crucial role. In this paper we consider one-dimensional (1D) special relativistic radiation hydrodynamics by solving the Boltzmann equation for radiative transfer. The structure of a radiative shock is calculated for a number of shock tube problems, including strong shock waves, and relativistic- and radiation-dominated cases. Calculations are performed using an iterative technique that consistently solves the equations of relativistic hydrodynamics and relativistic comoving radiative transfer. A comparison of radiative transfer solutions with the Eddington approximation and the M1 closure is made. A qualitative analysis of moment equations for radiation is performed and the conditions for the existence of jump discontinuity for non-relativistic cases are investigated numerically.

Subject headings: radiative transfer-relativistic processes-shock waves
\end{abstract}

\section{INTRODUCTION}

There are a number of topical high-energy astrophysical phenomena in which radiative transfer occurs in moving media: supernovae, gamma-ray bursts (GRBs), jets from active galactic nuclei, and collapsars. In all of these phenomena, radiative transfer can play a crucial role in the dynamics and should be included in simulations leading to the identification of observation signatures.

One of the most striking examples of phenomena of this type is supernova shock breakout. It produces a bright flash, caused by a shock wave emerging on the surface of the star after the phase of collapse or thermonuclear explosion in the interior. Recent detections of supernova shock breakouts (Schawinski et al. 2008; Gezari et al. 2008; Soderberg et al. 2008) require more accurate theoretical models, which are usually constructed numerically in non-relativistic treatment (see e.g. Klein \& Chevalier (1978); Ensman \& Burrows (1992); Kelly \& Korevaar (1995); Blinnikov et al. (1998, 2000); Katz et al. (2010); Tominaga et al. (2011); Katz et al. (2012); Sapir et al. (2013); Sapir \& Halbertal (2014)). Our previous study of supernova shock breakout phenomena (Tolstov et al. 2013) revealed the importance of accurate light curves of Type Ibc supernovae, where the velocity of matter at the epoch of shock breakout becomes mildly relativistic. The shock wave can reach highly relativistic velocities in exploding white dwarfs and hypernovae. Better descriptions of supernova shock breakout phenomena and the connection to supernovae with GRBs require a selfconsistent solution of relativistic radiation hydrodynamics and detailed study of shock wave structure.

In this paper we consider the problem of the

* E-mail: alexey.tolstov@ipmu.jp

5 Hamamatsu Professor. structure of shock waves that are coupled with radiation at an arbitrary velocity of matter for a one-dimensional plane stationary shock. There are many papers related to the shock wave structure problem (Zeldovich 1957; Raizer 1957; Belokogne 1959; Imshennik \& Morozov 1964; Morozov 1971; Belokogne 1972a; Weaver 1976; Chapline \& Granik 1984; Bouquet et al. 2000; Drake 2007; Coulombel \& Lafitte 2009; Kraiko 2011; Vavtet et al. 2013), but in all of them the non-relativistic motion of the fluid $(v / c \ll 1)$ or approximations of radiation field are considered. A number of papers have been published where the shock wave structure is calculated numerically for relativistic velocities of matter (Farris et al. 2008; Zanotti et al. 2011; Fragile et al. 2012; Sadowski et al. 2013; Takahashi et al. 2013; | McKinney et al.| 2014), but they are based on the solution of radiation moments equations using a closure condition. In some cases this approach provides a relevant solution, but sometimes it is difficult to estimate the relevance of the closure condition, so the exact solution of kinetic equation is required. To resolve this uncertainty and to estimate how accurate the previous studies are, we take into consideration the radiative transfer equation. This approach eliminates the closure condition and helps to clarify the role of radiation in the shock wave structure at various flow velocities.

As a striking example of the influence of radiative transfer on the structure of a radiative shock wave we will consider the phenomenon of the disappearance of shock wave jump discontinuity (viscous jump) in the radiation-dominated flow for non-relativistic velocities. The disappearance of the shock wave jump discontinu-

${ }^{6}$ Note that the name (Belokogne) is often spelled (Belokon'). 
ity occurs when the downstream ratio of the radiation pressure $p_{r}$ to the pressure of the gas $p_{g}$ rises above a critical value $\left(p_{r} / p_{g}\right)_{c r}$. The previous theoretical estimations provide values of the critical downstream ratio from $\left(p_{r} / p_{g}\right)_{c r} \simeq 4.45$ (Belokogne 1959; Weaver 1976) to $\left(p_{r} / p_{g}\right)_{c r} \simeq 8.5$ in more accurate considerations of radiation moment equations in the Eddington approximation (Imshennik \& Morozov 1964). The critical ratio $\left(p_{r} / p_{g}\right)_{c r}$ seems to be very sensitive to the closure condition and our numerical simulations and analytic estimations provide its value more accurately.

\section{STATEMENT OF THE PROBLEM}

The problem of radiative transfer in moving media in a general formulation leads to the solution of the system of the relativistic radiation hydrodynamics equations coupled with the relativistic radiative transfer equation. In this paper we consider one-dimensional (1D), plane, and stationary shock waves. Due to the complexity of the problem a number of simplifications are introduced: Kirchhoffs law of thermal radiation, the graybody opacity law, zero value scattering opacity, and the equation of state for an ideal gas.

Thus, the complete system of equations describing the shock wave structure consists of the equation of state, the equations of radiation hydrodynamics, and the equation of radiative transfer:

$$
n p=\rho \epsilon,
$$

where $n$ is the polytropic index, $\epsilon$ - specific internal energy,

$$
\begin{gathered}
\gamma \beta \rho=U_{1}, \\
\gamma^{2} \beta^{2}[\rho+(n+1) p]+p+P=U_{2}, \\
\gamma^{2} \beta[\rho+(n+1) p]+F=U_{3}, \\
\gamma\left(\mu_{0}+\beta\right) \frac{\partial I_{0}\left(\mu_{0}\right)}{\partial x}-\gamma^{3}\left(1-\mu_{0}^{2}\right)\left(\mu_{0}+\beta\right) \frac{d \beta}{d x} \frac{\partial I_{0}\left(\mu_{0}\right)}{\partial \mu_{0}}+ \\
+4 \gamma^{3} \mu_{0}\left(\mu_{0}+\beta\right) \frac{d \beta}{d x} I_{0}\left(\mu_{0}\right)=\eta_{0}-\chi_{0} I_{0}\left(\mu_{0}\right) .
\end{gathered}
$$

Here the density of the matter $\rho$ and the pressure $p$ in the equations are measured in the fluid frame. The radiative transfer equation is written in the comoving frame and the dependence of the specific intensity $I_{0}$ from the space variable is suppressed in the notation. The specific intensity $I_{0}$ and lab-frame radiation moments $E, F, P$ are related as:

$$
\begin{aligned}
\left\{E_{0}, F_{0}, P_{0}\right\} & =\int_{-1}^{1} I_{0}\left(\mu_{0}\right) \mu^{k} d \mu_{0}, k=0 \ldots 2, \\
E & =\gamma^{2}\left(E_{0}+2 \beta F_{0}+\beta^{2} P_{0}\right), \\
F & =\gamma^{2}\left(\left(1+\beta^{2}\right) F_{0}+\beta\left(E_{0}+P_{0}\right)\right), \\
P & =\gamma^{2}\left(P_{0}+2 \beta F_{0}+\beta^{2} E_{0}\right) .
\end{aligned}
$$

The equation of radiative transfer in the comoving frame (5) (Mihalas 1980) after integration over angle $\mu_{0}$ can be replaced by the radiation moments equations coupled with a closure condition (see Appendix A for details):

$$
\begin{gathered}
\frac{d F}{d x}=\frac{\kappa U_{1}}{\beta}\left(a_{R} T^{4}+\right. \\
\left.+\frac{\beta F\left(2-f-\beta^{2}\right)-P\left(1-f \beta^{2}\right)}{f-\beta^{2}}\right), \\
\frac{d P}{d x}=\frac{\kappa U_{1}}{\beta}\left(\left(a_{R} T^{4}+P\right) \beta-F\right), \\
P_{0}=f E_{0}
\end{gathered}
$$

Equations (10, 12) are easier to solve in comparison to the radiative transfer equation (5), but the choice of the Eddington factor $f$ in general case is not obvious. To compare the solution of the radiative transfer equation below we will consider a couple of approximations of the Eddington factor:

a). Eddington approximation (optically thick medium)

$$
f=\frac{1}{3}
$$

b). M1 closure (Minerbo 1978; Levermore 1984; Dubroca \& Feugeas 1999) (combines approximations for optically thick and optically thin media)

$$
f=\frac{1}{3}\left[5-2 \sqrt{4-3\left(\frac{F_{0}}{P_{0}}\right)^{2}}\right]
$$

\section{NUMERICAL SOLUTION AND TESTS}

To investigate how the radiative transfer affects the shock wave structure we consider a number of shock tube tests that are widely used in relativistic radiation hydrodynamics (Farris et al. 2008; Zanotti et al. 2011; Fragile et al. 2012; Sadowski et al.|2013; Takahashi et al. 2013; McKinney et al. 2014). These test data are summarized in Table 1

A number of closure conditions are used: the Eddington approximation, the M1 closure and the exact solution of radiative transfer problem.

We use RADA code (Tolstov \& Blinnikov 2003; Tolstov 2010) to solve the radiative transfer equation in the comoving frame (5). The code is based on the method of characteristics and can be applied to the motion of the fluid with arbitrary velocity. The code solves the Boltzmann form of the transport equation using the method of characteristics and can be applied to the motion of the fluid with an arbitrary velocity. The code is multigroup, but because we consider a gray medium, no spectra details are calculated. Hydro equations are solved semi-analytically (see Appendix A for details). The numerical space grid used for radiative transfer is independent of the hydro grid and is based on an adaptive algorithm to improve the performance of the characteristics method and resolve the discontinuities of radiation quantities. The hydro equation and the radiative transfer equation are solved by an iterative algorithm. The radiation moments equations (10,11) are integrated by Dormand-Prince method with adaptive step.

In all the tests, following Farris et al. (2008), we use dimensionless quantities to make a comparison with already published results. The upstream gas density $\rho_{L}$ 


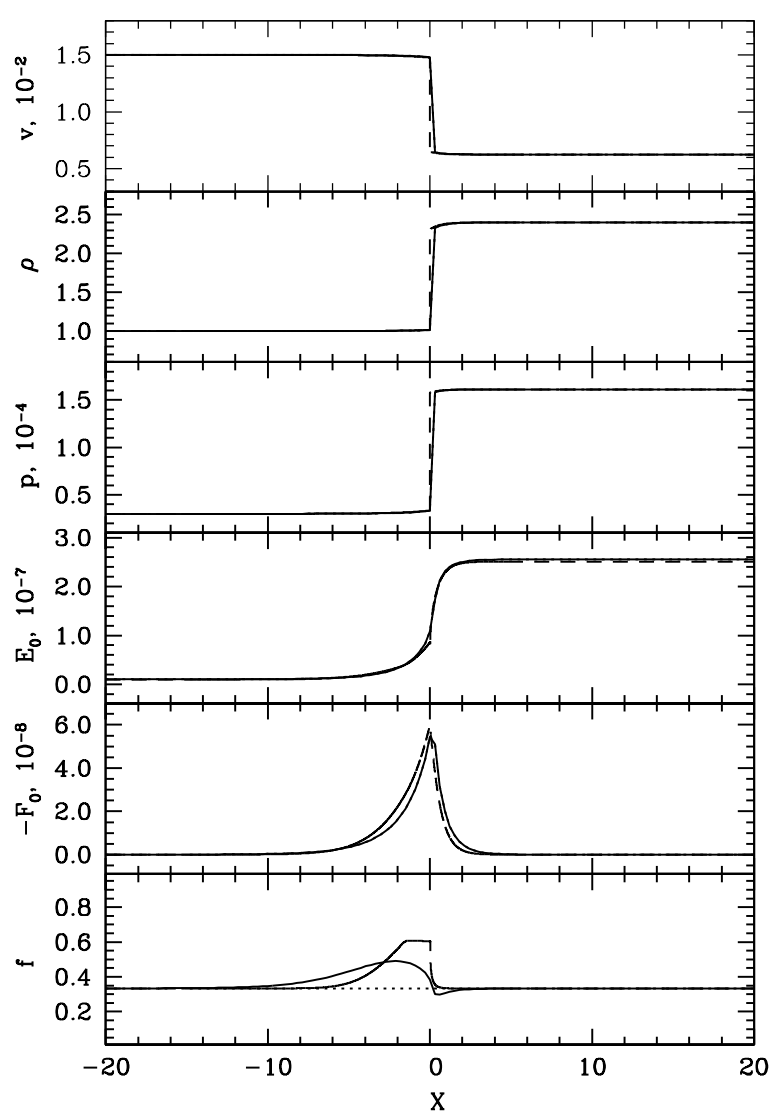

Figure 1. Profiles of $\beta, \rho, p, E_{0}, F_{0}, f$ for test $\mathrm{f} 1$ at various radiation closure conditions. The dotted line is the Eddington approximation; the dashed line is the M1 closure; and the solid line is the exact solution of the radiative transfer equation.

is set to unity in all the tests. The choice of the other upstream quantities $v_{L}, p_{L}, E_{L}$ depends on the case to be considered. For a non-relativistic shock $v \ll 1$, and for a relativistic shock $v$ is close to unity. The values of pressure $p_{L}$ and radiation energy $E_{L}$ determine how strong the shock is and whether or not the shock is radiationdominant. In order to find all downstream physical quantities and to construct a shock tube configuration the nonlinear equation is solved (see Appendix A).

In this dimensionless approach the density in CGS units is related to dimensionless density as follows: $\rho_{C G S}=\rho\left[a_{R}\left(\frac{p_{L}}{\rho_{L}} \frac{m}{k_{B}} c^{2}\right)^{4} /\left(E_{L} c^{2}\right)\right]$, where $a_{R}$ is the radiation constant, $m$ is the mean mass of baryons in the fluid, $k_{B}$ is Boltzmann's constant and $c$ is the speed of light.

\subsection{Non-relativistic strong shock}

In this test a strong, gas-pressure dominated, nonrelativistic shock is propagating into a cold gas. The exact solution of radiative transfer and the M1 closure provide much higher values of the Eddington factor $f$ near the shock front than the Eddington closure (Fig [1). Nevertheless this does not significantly change the hydro and radiation profiles. The high value of the Eddington factor in the upstream has almost no effect on the gas-pressure dominated, cold medium.

\subsection{Mildly-relativistic strong shock}

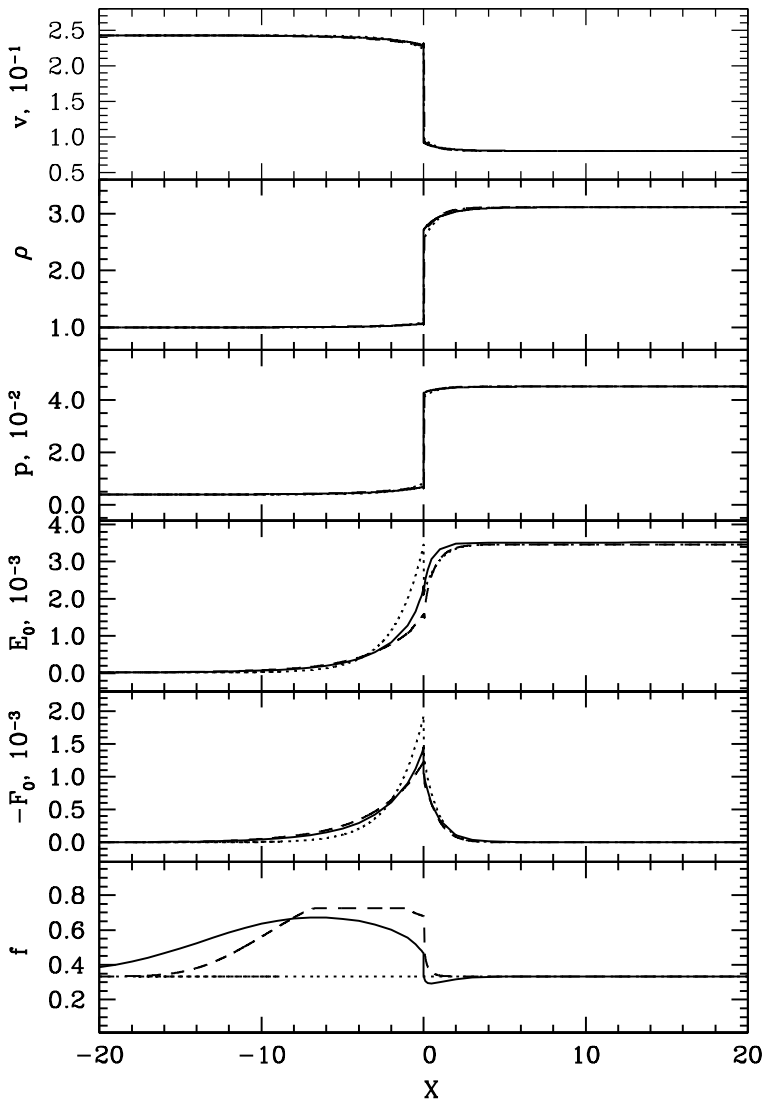

Figure 2. Profiles of $\beta, \rho, p, E_{0}, F_{0}, f$ for test $\mathrm{f} 2$ at various radiation closure conditions. The dotted line is the Eddington approximation; the dashed line is the M1 closure; and the solid line is the exact solution of the radiative transfer equation.

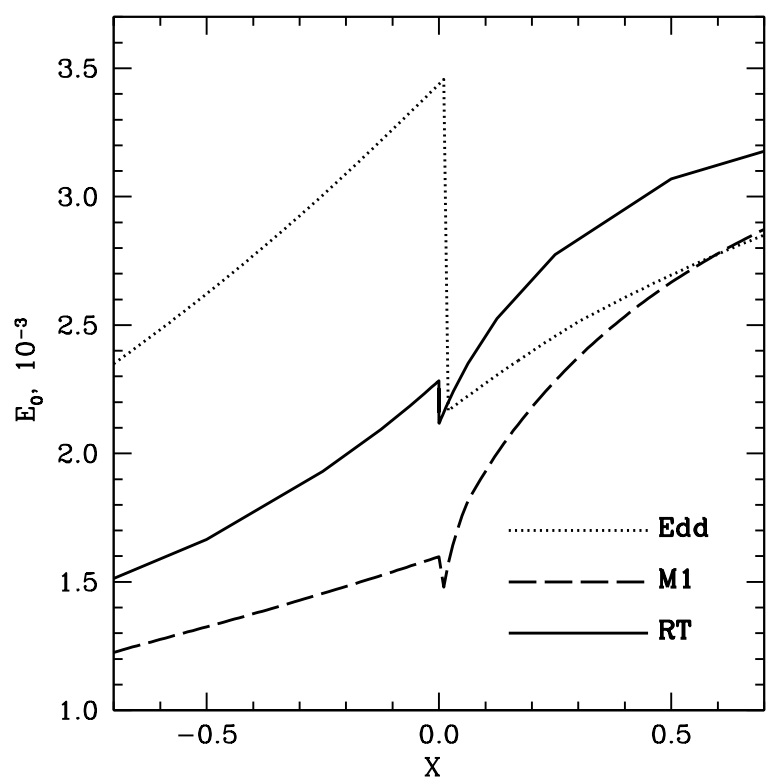

Figure 3. Radiation energy density ptofile near the discontinuity for test f2. The dotted line is the Eddington approximation; the dashed line is the M1 closure; and the solid line is the exact solution of the radiative transfer equation. 
Table 1

Test Parameters for Left(L) and Right(R) States, $\rho_{L}=1$.

\begin{tabular}{llllllllll}
\hline \hline Test & $\mathrm{n}$ & $v_{L}$ & $p_{L}$ & $E_{L}$ & $\rho_{R}$ & $v_{R}$ & $p_{R}$ & $E_{R}$ & $\kappa^{a}$ \\
\hline $\mathrm{f} 1$ & 1.5 & $1.50 \cdot 10^{-2}$ & $3 \cdot 10^{-5}$ & $1.0 \cdot 10^{-8}$ & 2.40 & $6.25 \cdot 10^{-3}$ & $1.61 \cdot 10^{-4}$ & $2.5 \cdot 10^{-7}$ & 0.4 \\
$\mathrm{f} 2$ & 1.5 & $2.43 \cdot 10^{-1}$ & $4 \cdot 10^{-3}$ & $2.0 \cdot 10^{-5}$ & 3.11 & $8.01 \cdot 10^{-2}$ & $4.51 \cdot 10^{-2}$ & $3.5 \cdot 10^{-3}$ & 0.2 \\
$\mathrm{f} 3$ & 2 & $9.95 \cdot 10^{-1}$ & 60 & 2.0 & 7.99 & 0.78 & $2.34 \cdot 10^{3}$ & $1.1 \cdot 10^{3}$ & 0.3 \\
$\mathrm{f} 4$ & 1.5 & $5.68 \cdot 10^{-1}$ & $6 \cdot 10^{-3}$ & $1.8 \cdot 10^{-1}$ & 3.65 & 0.18 & $3.58 \cdot 10^{-2}$ & 1.3 & 0.08 \\
\hline \hline
\end{tabular}

\begin{tabular}{lllll}
\hline \hline Test & $\left(p_{r} / p_{g}\right)_{L}$ & $\left(p_{r} / p_{g}\right)_{R}$ & $\left(p_{R} / p_{L}\right)$ & $\left(E_{R} / E_{L}\right)$ \\
\hline f1 & $1.11 \cdot 10^{-4}$ & $5.19 \cdot 10^{-4}$ & 5.37 & $2.5 \cdot 10^{1}$ \\
f2 & $1.67 \cdot 10^{-3}$ & $2.55 \cdot 10^{-2}$ & 11.2 & $1.7 \cdot 10^{2}$ \\
f3 & $1.11 \cdot 10^{-2}$ & $1.61 \cdot 10^{-1}$ & 39.0 & $5.6 \cdot 10^{2}$ \\
f4 & 10 & $1.20 \cdot 10^{1}$ & 5.98 & 7.2 \\
\hline \hline
\end{tabular}

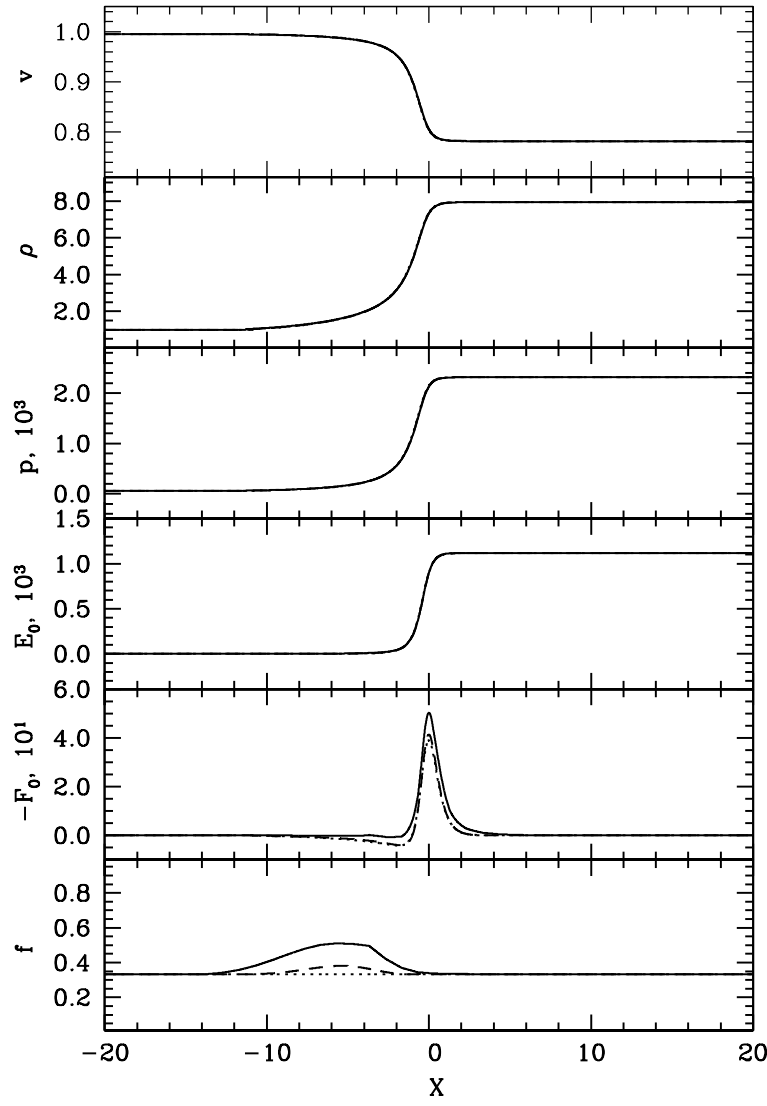

Figure 4. Profiles of $\beta, \rho, p, E_{0}, F_{0}, f$ for test $\mathrm{f} 3$ at various radiation closure conditions. The dotted line is the Eddington approximation; the dashed line is the M1 closure; and the solid line is the exact solution of the radiative transfer equation.

The junction conditions for the radiation variables are the continuity of the radiation flux $F$ and the radiation pressure $P$. But in the comoving frame these variables can become discontinuous. This test reveals discontinuity not only in hydrodynamic quantities but in the comoving radiation energy density and the radiation energy flux (Fig. 2). The jump of the radiation energy density $E_{0}$ can roughly be estimated by a simplified assumption of an optically thick medium and a velocity profile as a step function at the discontinuity region. Using an invariant frequency-integrated intensity $I / \nu^{4}$ for

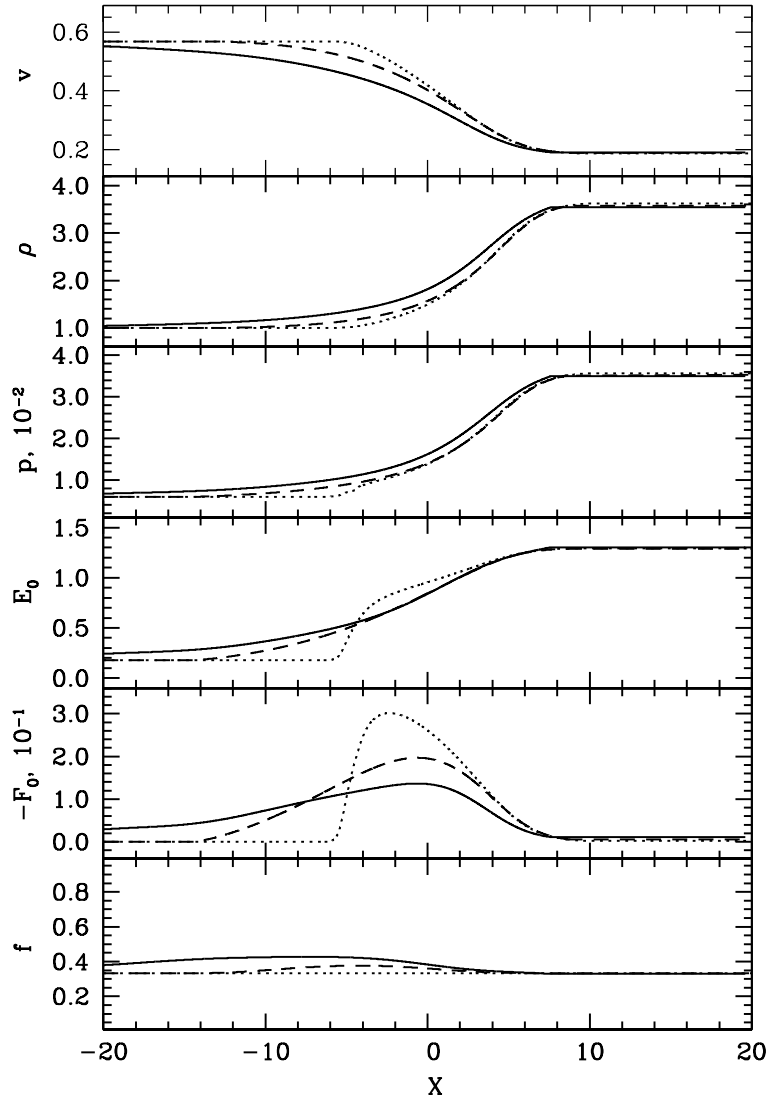

Figure 5. Profiles of $\beta, \rho, p, E_{0}, F_{0}, f$ for test $\mathrm{f} 4$ at various radiation closure conditions. The dotted line is the Eddington approximation; the dashed line is the M1 closure; and the solid line is the exact solution of the radiative transfer equation.

the radiation energy density jump $\Delta E_{0, d}$ we find:

$$
\begin{gathered}
\Delta E_{0, d}=E_{L, d}-E_{R, d} \approx \\
\approx \frac{1}{2}\left[E_{R} \int_{\beta_{L}}^{1} \frac{d \mu}{(1-\mu \beta)^{4}}+E_{L} \int_{-\beta_{L}}^{1} d \mu\right]- \\
-\frac{1}{2}\left[E_{L} \int_{-\beta_{R}}^{1} \frac{d \mu}{(1-\mu \beta)^{4}}+E_{R} \int_{\beta_{R}}^{1} d \mu\right], \\
\beta=\frac{\beta_{L}-\beta_{R}}{1-\beta_{L} \beta_{R}} .
\end{gathered}
$$

Substituting $\beta_{L}$ and $\beta_{R}$ from Table I one can estimate the upper limit of the jump as $\Delta E_{0, d} \simeq 3.5 \cdot 10^{-4}$, which 
is in good agreement with our numerical calculation, yet several times lower than in the Eddington approximation (Fig. 3). The M1 closure gives a much better approximation of relativistic transformations for this test.

Calculating this kind of discontinuity requires a fine space grid for radiative transfer and we use an adaptive grid to increase the number of points near the discontinuity region. We note that the same discontinuity can be resolved for Test f1 as well, but it is negligibly small due to non-relativistic velocities.

\subsection{Highly-relativistic wave}

This is a gas-dominated test, but no discontinuity is observed (Fig. 4) due to hot upstream gas. Similar to test $\mathrm{f1}$, no significant difference between the various closure conditions is revealed in the profiles. The Eddington factor in the upstream cannot affect radiation profiles because of high radiation energy density in the downstream.

\subsection{Radiation-pressure dominated, mildly-relativistic wave}

This test is radiation-dominated, but the shock wave is not strong and the radiation energy density in the downstream is only several times higher than that in the upstream. This fact leads to a larger dependence of radiation hydro profiles on closure conditions (Fig. 5). Both the hydro and radiation profiles become smoother for the M1 closure in comparison with the Eddington approximation, and are even smoother in the exact calculation of the radiative transfer. Thus in this test the solution is quite sensitive to the Eddington factor value.

\section{QUALITATIVE ANALYSIS}

One can see that in some tests from the previous section we find discontinuity, but in others all of the profiles are smooth. The critical downstream radiation-togas pressure ratio $\left(p_{r} / p_{g}\right)_{c r}$, at which the discontinuity disappears in nonrelativistic consideration, has been investigated previously. In consideration of shock jump conditions the ratio $\left(p_{r} / p_{a}\right)_{c r} \simeq 4.45$ for monoatomic gas (Belokogne (1959); Weaver $(1976)$; see also the detailed study in Belokogne (1972a) and the more correct original paper (Belokogne 1972b)). Using a more sophisticated approach for the Eddington approximation $\left(\left(p_{r} / p_{g}\right)_{c r} \simeq 8.5\right.$, (Imshennik \& Morozov 1964)). In more a general formulation $p_{r} / p_{q}=2+(3+2 n)^{1 / 2}$ (Belokogne (1972a), see also Weaver \& Chapline (1974) for a relativistic case), but in this paper we limit our consideration by the polytropic index $n=1.5$.

Using the solution of the radiative transfer problem the exact value of this ratio can be calculated using our code.

In all of the calculations of the critical ratio the upstream gas is supposed to be cold, its pressure is supposed to be low and the fluid velocities should be nonrelativistic

Our analysis is based on the approach described in Imshennik \& Morozov (1964) paper. . In that paper, the approximate solution of the problem and a qualitative analysis of equations (10,11) in the phase plane are performed in the Eddington approximation. This approach gives the critical ratio $\left(p_{r} / p_{g}\right)_{c r} \simeq 8.5$ (Imshennik \& Morozov 1964).

In this paper we extend the phase plane analysis to arbitrary velocities (see Appendex A), take radiative trans-

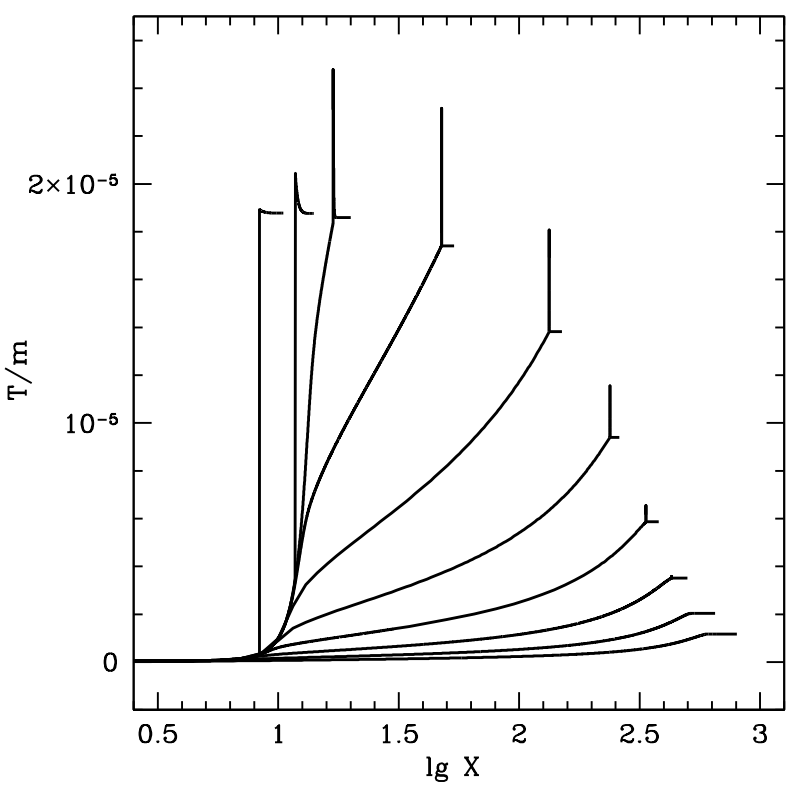

Figure 6. Temperature profile of the shock front in the presence of radiation in the Eddington approximation closure. The radiation influence $p_{r} / p_{g}$ increases from the left to the right profile (Table I).

fer into consideration, and construct a number of tests to analyze the shock wave structure for low-pressure, cold upstream gas, and specify the value of the critical ratio in the exact solution of the radiative transfer equation.

The tests we have constructed (Table II) provide a set of shock tube configurations from classical shock, with discontinuity between the radiation-dominated shock waves up to the downstream radiation-to-gas pressure ratio $p_{r} / p_{g} \simeq 10$. Upstream density, velocity, and pressure are the same for all of the tests, radiation energy density is a variable parameter, and upstream pressure and temperature are negligible in comparison with downstream pressure and temperature.

Our modeling of the shock front using the Eddington closure reproduces Zeldovich spike behavior and the results of Imshennik \& Morozov (1964) paper: i.e., the disappearance of the shock jump at $p_{r} / p_{g} \simeq 8.5$ (Fig. 6). At $p_{r} / p_{g}>5$ the peak temperature differs from the downstream temperature by a fraction of a percent and there is not much difference in the shock wave structure between the solutions with different closure conditions.

We also performed the calculation of the profiles with the M1 closure, but the result for the strong shock is almost the same as for the Eddington closure (similar to tests f1-f3 in the previous section) because radiation flux $F_{0}$ is comparable with radiation density $E_{0}$ only in the part of the shock front where the temperature is relatively low (see Figure 77).

Calculations with the radiation transfer equation do not significantly change the profiles for the same reason: a high Eddington factor affects only the low-energy part of the temperature profiles (see Figures 8, 9).

The critical ratio in the Eddington approximation $\left(p_{r} / p_{g}\right)_{c r} \simeq 8.5$ does not correspond to the value $\left(p_{r} / p_{g}\right)_{c r} \simeq 4.45$ found by Belokogne (1959). The approach described by Imshennik \& Morozov (1964) seems to be more accurate because the complete system of ra- 
Table 2

Test Parameters at $v_{L}=0.01, \rho_{L}=1, p_{L}=3 \cdot 10^{-8}, n=1.5$.

\begin{tabular}{llllllllll}
\hline \hline Test & $\lg E_{L}$ & $\rho_{R}$ & $v_{R}, 10^{-3}$ & $p_{R}, 10^{-5}$ & $E_{R}$ & $\left(p_{r} / p_{g}\right)_{L}$ & $\left(p_{r} / p_{g}\right)_{R}$ & $\left(p_{R} / p_{L}\right)$ & $\left(E_{R} / E_{L}\right)$ \\
\hline s1 & -19 & 3.994 & 2.503 & 7.499 & $1.53 \cdot 10^{-8}$ & $1.11 \cdot 10^{-12}$ & $6.81 \cdot 10^{-5}$ & 2500 & $1.53 \cdot 10^{11}$ \\
s2 & -18 & 3.996 & 2.502 & 7.496 & $1.52 \cdot 10^{-7}$ & $1.11 \cdot 10^{-11}$ & $6.79 \cdot 10^{-4}$ & 2498 & $1.52 \cdot 10^{11}$ \\
s3 & -17 & 4.013 & 2.491 & 7.462 & $1.47 \cdot 10^{-6}$ & $1.11 \cdot 10^{-10}$ & $6.59 \cdot 10^{-3}$ & 2487 & $1.47 \cdot 10^{11}$ \\
s4 & -16 & 4.143 & 2.413 & 7.212 & $1.13 \cdot 10^{-5}$ & $1.11 \cdot 10^{-9}$ & $5.23 \cdot 10^{-2}$ & 2404 & $1.13 \cdot 10^{11}$ \\
s5 & -15 & 4.569 & 2.188 & 6.314 & $4.50 \cdot 10^{-5}$ & $1.11 \cdot 10^{-8}$ & $2.37 \cdot 10^{-1}$ & 2104 & $4.50 \cdot 10^{10}$ \\
s6 & -14 & 5.183 & 1.929 & 4.869 & $9.61 \cdot 10^{-5}$ & $1.11 \cdot 10^{-7}$ & $6.58 \cdot 10^{-1}$ & 1623 & $9.61 \cdot 10^{9}$ \\
s7 & -13 & 5.764 & 1.734 & 3.383 & $1.46 \cdot 10^{-4}$ & $1.11 \cdot 10^{-6}$ & 1.44 & 1127 & $1.46 \cdot 10^{9}$ \\
s8 & -12 & 6.213 & 1.609 & 2.178 & $1.86 \cdot 10^{-4}$ & $1.11 \cdot 10^{-5}$ & 2.85 & 726 & $1.86 \cdot 10^{8}$ \\
s9 & -11 & 6.519 & 1.533 & 1.330 & $2.14 \cdot 10^{-4}$ & $1.11 \cdot 10^{-4}$ & 5.36 & 443 & $2.14 \cdot 10^{7}$ \\
s10 & -10 & 6.714 & 1.489 & 0.785 & $2.31 \cdot 10^{-4}$ & $1.11 \cdot 10^{-3}$ & 9.83 & 261 & $2.31 \cdot 10^{6}$ \\
\hline \hline
\end{tabular}

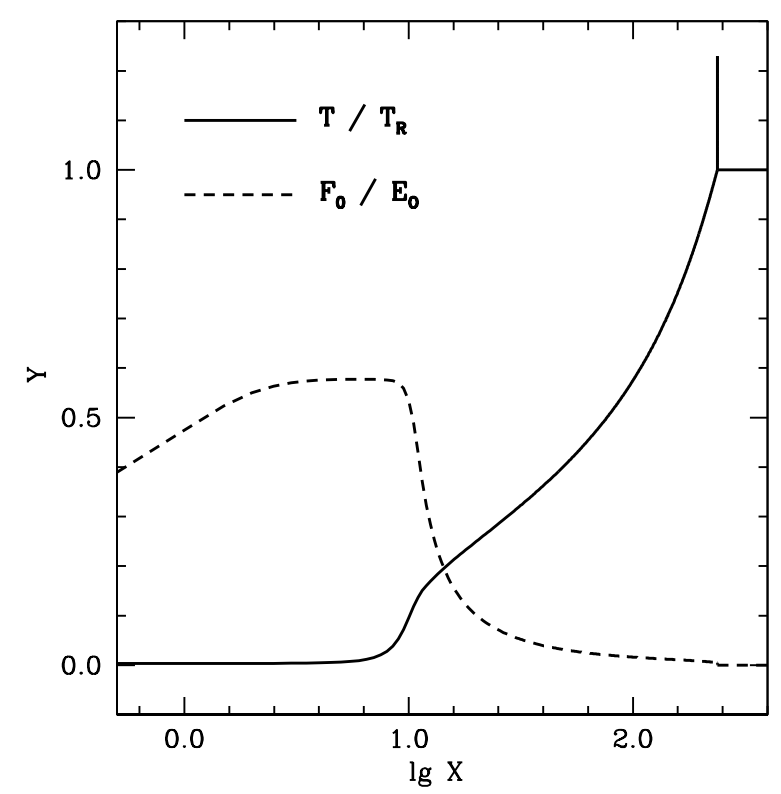

Figure 7. Normalized temperature and radiation flux for test s4 (Table 1) in solution with the M1 closure.

diation hydrodynamics equations is considered and as shown in their paper, the Eddington approximation is more reliable in comparison with the diffusion approximation. But the question remains regarding how rigorous the Eddington approximation is in comparison with the exact solution of a kinetic equation.

Using our code and a semi-analytic approach (see Appendix A we calculated the critical ratio by solving the complete system of radiation hydrodynamics equations with no closure condition. The critical ratio in this case is $\left(p_{r} / p_{g}\right)_{c r} \simeq 4.5$ we calculated the critical ratio by solving the complete system of radiation hydrodynamics equations with no closure condition. The critical ratio in this case is Belokogne (1959). In contrast to the Eddington approximation, in our calculation the Eddington factor is not constant. In the discontinuity region its value is lower and consequently, lower radiation pressure is required to reach a continuous solution. The accuracy of our iterative method is high enough to estimate the critical ratio and compare it with the previous studies, but further improvements of the numerical approach are needed both for an exact calculation of $\left(p_{r} / p_{g}\right)_{c r}$ and for a consideration of relativistic flows.

The diffusion approximation is widely used for the cal-

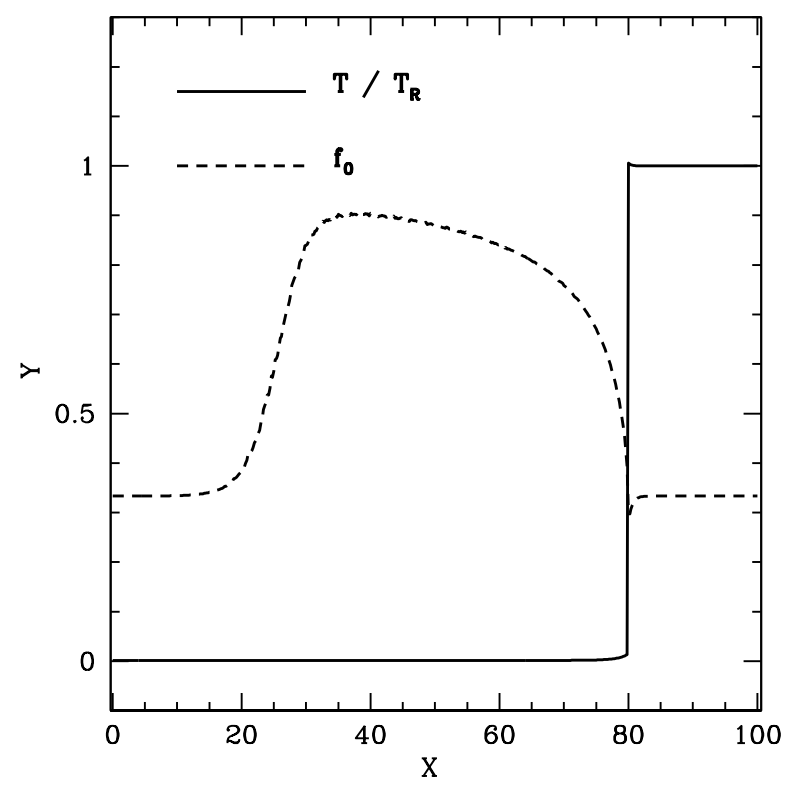

Figure 8. Normalized temperature and Eddington factor for test s1 (Table II) in solution with the radiative transfer equation.

culation of the critical ratio $\left(p_{r} / p_{g}\right)_{c r} \simeq 4.45$ by consideration of the "isothermal jump" (Landau \& Lifshitz 1959; Belokogne 1959; Bouquet et al. 2000). But the "isothermal jump" is a consequence of the mathematical approximation where the flow is proportional to the temperature gradient. This approximation eliminates the temperature jump at the shock. But the temperature jump occurs due to nonequilibrium radiation (see Zeldovich \& Raizer (1966) and Fig. 6) and the critical ratio becomes higher in more accurate Eddington approximations $\left(\left(p_{r} / p_{g}\right)_{c r} \simeq 8.5\right)$ (Imshennik \& Morozov 1964). Our calculations show that both the Eddington approximation and the M1 closure disregard the contribution of $d f / d F, d f / d P$ terms in the calculation of the critical ratio and overestimate it.

In the case where we cannot neglect the energy of the upstream gas the critical ratio will be lower than its maximum values (see Fig. 10 and a more detailed investigation by Imshennik (1962)). We found a similar situation in test f3. However, test f3 is highly relativistic and requires a separate, more accurate investigation.

\section{CONCLUSIONS}

Our investigation shows that assuming graybody opacity and the equation of state for an ideal gas in non- 


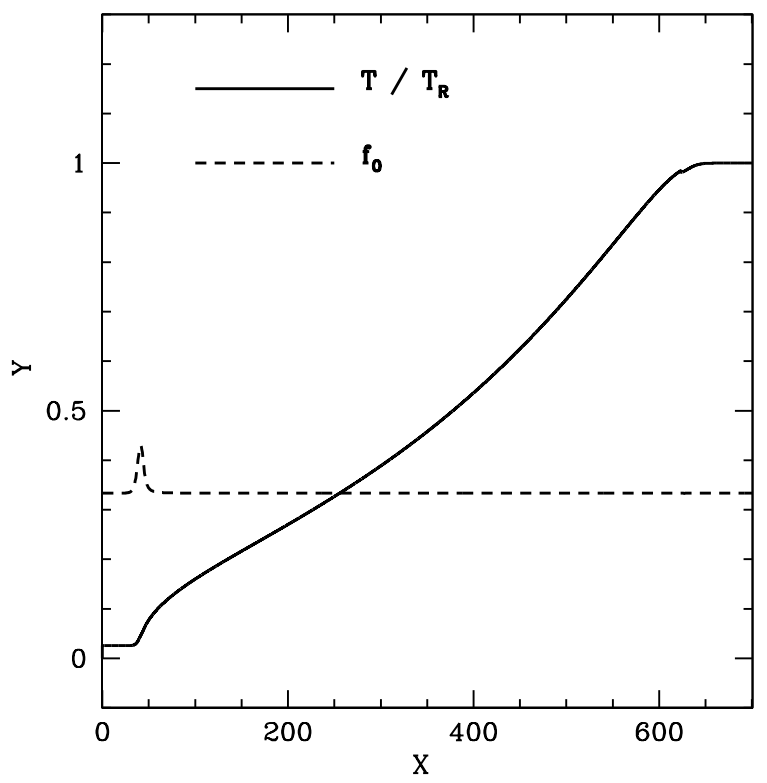

Figure 9. Normalized temperature and Eddington factor for test s10 (Table II) in solution with the radiative transfer equation.

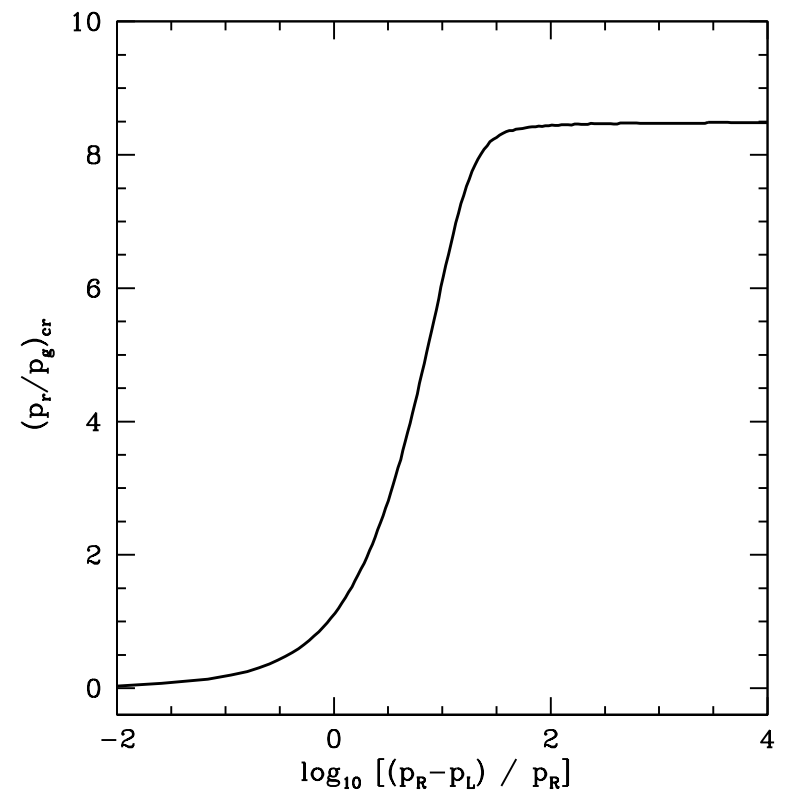

Figure 10. Downstream critical radiation-to-gas pressure ratio $\left(p_{r} / p_{g}\right)_{c r}$ depending on the fractional pressure jump in the Eddington approximation closure.

relativistic strong shock waves has a good accuracy in calculating the shock front structure, regardless of the dominance of radiation. This is not the case for relatively weak shocks where radiative transfer can be accurately taken into account to have reliable calculations. Radiative transfer can affect the gas in the head part of the shock front and closure approximations cannot already be used for accurate calculations of radiation fluxes.

The analysis of the criteria for the disappearance of shock wave discontinuity in radiation-dominated media for strong shock waves shows that an accurate approach for calculating the Eddington factors provides the result $\left(p_{r} / p_{g}\right)_{c r} \simeq 4.5$. This value is close to the results that were originally derived by Belokogne (1959): $\left(p_{r} / p_{g}\right)_{c r} \simeq$ 4.45 for monoatomic gases. Approximations of Eddington factors (e.g., the Eddington, the M1 closure) give the critical ratio $\left(p_{r} / p_{g}\right)_{c r} \simeq 8.5$ Imshennik \& Morozov 1964), but for strong shocks radiation and hydro profiles are not significantly affected. The variations of the equation of state have a greater influence on the profiles (Belokogne 1972a).

A complete consideration of the shock wave structure requires an analysis of relativistic and ultra-relativistic cases along with scattering in details and will be discussed separately. The qualitative analysis we have used for shock wave structure can be extended to relativistic cases, but it requires a higher accuracy for the solution of the integral-differential equation of radiative transfer. In our preliminary calculations of relativistic flows for both the radiative transfer solutions and radiation moment closure, the critical downstream radiation-to-gas pressure ratio $\left(p_{r} / p_{q}\right)_{c r}$ becomes larger with increasing velocity of the shock wave, due to a decreasing of the preheated region. But at highly relativistic velocities the influence of the Eddington factor approximation on the shock wave structure becomes more significant and the usage of a pre-defined radiation moment closure may lead to incorrect results.

Our approach in the modeling of the shock wave structure at arbitrary velocities of the matter is an important step toward realistic simulations of shock breakouts where the velocity of matter becomes highly relativistic. This includes simulations of hypernovae, superluminous supernovae, Type Ibc supernovae, exploding white dwarfs, GRBs, and their afterglows.

\section{ACKNOWLEDGMENTS}

This research is supported by the World Premier International Research Center Initiative (WPI Initiative), MEXT, Japan. The work of S.B. (general formulation of the problem and numerical algorithms) is supported by the Russian Science Foundation Grant No. 14-12-00203. KN and AT are supported by the Grant-in-Aid for Scientific Research of the JSPS (23224004), Japan.

\section{REFERENCES}

Andronov, A. A., Vitt, A. A., \& Khaikin, S. W. 1987, Theory of Oscillators (New York: Dover)

Belokogne, V. A. 1959, Soviet Physics - JETP, 9, 235

—. 1972a, Soviet Physics Doklady, 17, 132

—. 1972b, Doklady Akad. Nauk SSSR [in Russian], 202, 1296

Blinnikov, S., Lundqvist, P., Bartunov, O., Nomoto, K., \& Iwamoto, K. 2000, ApJ, 532, 1132

Blinnikov, S. I., Eastman, R., Bartunov, O. S., Popolitov, V. A., \& Woosley, S. E. 1998, ApJ, 496, 454

Bouquet, S., Romain, T., \& Chieze, J. P. 2000, ApJS, 127, 245

Chandrasekhar, S. 1950, Radiative transfer.

Chapline, G. F., \& Granik, A. 1984, Physics of Fluids, 27, 1991

Coulombel, J.-F., \& Lafitte, P. 2009,

Communications in Computational Physics, 6, 1118

Drake, R. P. 2007, Physics of Plasmas, 14, 043301

Dubroca, B., \& Feugeas, J. 1999,

Academie des Sciences Paris Comptes Rendus Serie Sciences Mathematiques, Ensman, L., \& Burrows, A. 1992, ApJ, 393, 742

Farris, B. D., Li, T. K., Liu, Y. T., \& Shapiro, S. L. 2008, Phys. Rev. D, 78, 024023

Fragile, P. C., Gillespie, A., Monahan, T., Rodriguez, M., \& Anninos, P. 2012, ApJS, 201, 9 
Gezari, S., Dessart, L., Basa, S., et al. 2008, ApJ, 683, L131 Imshennik, V. S. 1962, Soviet Physics - JETP, 15, 167

Imshennik, V. S., \& Morozov, Y. I. 1964, Zh. Prikl. Mekh. Tekh. Fiz., 2, 8

Katz, B., Budnik, R., \& Waxman, E. 2010, ApJ, 716, 781

Katz, B., Sapir, N., \& Waxman, E. 2012, ApJ, 747, 147

Kelly, D. R. C., \& Korevaar, P. 1995, A\&A, 296, 418

Klein, R. I., \& Chevalier, R. A. 1978, ApJ, 223, L109

Kraiko, A. N. 2011,

Journal of Applied Mathematics and Mechanics, 75, 539

Landau, L. D., \& Lifshitz, E. M. 1959, Fluid mechanics

Levermore, C. D. 1984, J. Quant. Spec. Radiat. Transf., 31, 149

McKinney, J. C., Tchekhovskoy, A., Sadowski, A., \& Narayan, R. 2014, MNRAS, 441, 3177

Mihalas, D. 1980, ApJ, 237, 574

Minerbo, G. N. 1978, J. Quant. Spec. Radiat. Transf., 20, 541

Morozov, Y. I. 1971, Preprint N 66 [in Russian], IPM RAS

Raizer, Y. P. 1957, Soviet Physics - JETP, 5, 1242

Sapir, N., \& Halbertal, D. 2014, ApJ, 796, 145

Sapir, N., Katz, B., \& Waxman, E. 2013, ApJ, 774, 79

Sądowski, A., Narayan, R., Tchekhovskoy, A., \& Zhu, Y. 2013, MNRAS, 429, 3533

Schawinski, K., Justham, S., Wolf, C., et al. 2008,

Science, 321, 223
Soderberg, A. M., Berger, E., Page, K. L., et al. 2008, Nature, 453, 469

Takahashi, H. R., Ohsuga, K., Sekiguchi, Y., Inoue, T., \&

Tomida, K. 2013, ApJ, 764, 122

Tolstov, A. G. 2010, Astronomy Letters, 36, 109

Tolstov, A. G., \& Blinnikov, S. I. 2003,

Astronomy Letters, 29, 353

Tolstov, A. G., Blinnikov, S. I., \& Nadyozhin, D. K. 2013, MNRAS, 429, 3181

Tominaga, N., Morokuma, T., Blinnikov, S. I., et al. 2011, ApJS, 193, 20

Vaytet, N., González, M., Audit, E., \& Chabrier, G. 2013, J. Quant. Spec. Radiat. Transf., 125, 105

Weaver, T. A. 1976, ApJS, 32, 233

Weaver, T. A., \& Chapline, G. F. 1974, ApJ, 192, L57

Zanotti, O., Roedig, C., Rezzolla, L., \& Del Zanna, L. 2011, MNRAS, 417, 2899

Zeldovich, Y. B. 1957, Soviet Physics JETP, 5, 919

Zeldovich, Y. B., \& Raizer, Y. P. 1966, Physics of shock waves and high-temperature hydrodynamic phenomena (New York: Academic Press)

\section{APPENDIX}

\section{A. MOMENT EQUATIONS OF THE RADIATION FIELD}

For steady flow in plane geometry the radiation field is described as follows: (Mihalas 1980)

$$
\begin{aligned}
& 4 \pi \frac{d H}{d x}=q^{0}=\gamma\left(q_{0}^{0}+\beta q_{0}^{1}\right), \\
& \frac{4 \pi}{c} \frac{d K}{d x}=q^{1}=\gamma\left(\beta q_{0}^{0}+q_{0}^{1}\right),
\end{aligned}
$$

where $q^{\alpha}$ is a four-vector whose components specify the rate of momentum and energy exchange between radiation and matter,

$$
\begin{aligned}
q^{0} & =\int_{0}^{\infty} d \nu \oint d \omega[\eta(\mu, \nu)-\chi(\mu, \nu) I(\mu, \nu)] \\
q^{1} & =\int_{0}^{\infty} d \nu \oint d \omega[\eta(\mu, \nu)-\chi(\mu, \nu) I(\mu, \nu)] \mu \\
q_{0}^{0} & =4 \pi \int_{0}^{\infty}\left[\eta_{0}\left(\nu_{0}\right)-\chi_{0}\left(\nu_{0}\right) J_{0}(\nu)\right] d \nu_{0} \\
q_{0}^{1} & \left.=-4 \pi \int_{0}^{\infty} \chi_{0}\left(\nu_{0}\right) H_{0}(\nu)\right] d \nu_{0}
\end{aligned}
$$

where the subscript o relates to the comoving frame.

We assume a graybody form for all opacities

$$
\begin{gathered}
\chi_{0}\left(\nu_{0}\right)=\kappa \rho_{0}, \\
\kappa=\kappa_{a b s}+\kappa_{s c}=\kappa_{a b s},
\end{gathered}
$$

where $\kappa$ is frequency- independent opacity and $\rho_{0}$ is the rest mass energy density. Scattering opacity is understood to be zero. The thermal emissivity $\eta_{0}\left(\nu_{0}\right)$ and the absorption coefficient $\chi_{0}\left(\nu_{0}\right)$ are related by Kirchhoff's law $\eta_{0}=\chi_{0} B_{0}$ :

$$
\begin{aligned}
& q_{0}^{0}=4 \pi \chi_{0}\left(B_{0}-J_{0}\right)=\kappa \rho_{0}\left(4 \pi B_{0}-E_{0}\right), \\
& q_{0}^{1}=-4 \pi \chi_{0} H_{0}=-\kappa \rho_{0} F_{0},
\end{aligned}
$$

where $B$ is the intensity the in thermal equilibrium.

Substituting components of the four-vector in (A2), moment equations can be written in the following form:

$$
\begin{aligned}
& \frac{d F}{d x}=\frac{\kappa U_{1}}{\beta}\left(a_{R} T^{4}+\frac{\beta F\left(2-f-\beta^{2}\right)-P\left(1-f \beta^{2}\right)}{f-\beta^{2}}\right), \\
& \frac{d P}{d x}=\frac{\kappa U_{1}}{\beta}\left(\left(a_{R} T^{4}+P\right) \beta-F\right)
\end{aligned}
$$


where $a_{R}$ is the radiation constant and for temperature $T$ we have the following expression:

$$
T=\frac{m}{k_{B}} \frac{p}{\rho}=\frac{m}{k_{B}}\left(\frac{U_{3}-\gamma U_{1}-F}{U_{1} \gamma(n+1)}\right)
$$

where $m$ is the mean mass of baryons in the fluid, $k_{B}$ is Boltzmann's constant. Hereafter we set $k_{B}=1$.

The elimination of $p$ and $\rho$ from the radiation hydrodynamics equations gives the following equation for $\beta$ :

$$
H(\beta, F, P, n)=-U_{1} / \gamma-(n+1) \beta\left(U_{2}-P\right)+\left(1+n \beta^{2}\right)\left(U_{3}-F\right)=0 .
$$

Let us perform phase analysis in the $P F$ plane. After some algebra equilibrium points $F_{x}$ and $P_{x}$ of the system (1)-(2) can be found from the following algebraic equation

$$
H\left(\beta_{x}, 0,0, n\right)(1-n f)^{3}=\beta_{x} a_{R} m^{4}\left[\frac{f \gamma_{x} H\left(\beta_{x}, 0,0,1 / f\right)}{U_{1}}\right]^{4} .
$$

Finding $\beta_{x}$ from this equation, $F_{x}$ and $P_{x}$ can be determined from:

$$
\begin{gathered}
F_{x}=\frac{(1+f) \gamma_{x}^{2} H\left(\beta_{x}, 0,0, n\right)}{1-n f}, \\
P_{x} \beta_{x}=F_{x}-\beta_{x} a_{R}\left(T\left(\beta_{x}, F_{x}, n\right)\right)^{4} .
\end{gathered}
$$

The equation A15 has several roots, but only two of them have physical meaning with non-negative pressure. These roots can be used for the construction of shock tube configuration.

Let us write the equations (A12) in linear form in the neighbourhood of an equilibrium point:

$$
\begin{aligned}
& \frac{d F}{d x}=a(P, F)\left(P-P_{x}\right)+b(P, F)\left(F-F_{x}\right), \\
& \frac{d P}{d x}=c(P, F)\left(P-P_{x}\right)+d(P, F)\left(F-F_{x}\right),
\end{aligned}
$$

where coefficients are equaled to

$$
\begin{gathered}
\begin{aligned}
& a=\kappa U_{1}\left[\frac { a _ { R } T ^ { 3 } } { \beta ^ { 2 } } \left(4 \beta \frac{\partial T}{\partial P}\right.\right.\left.-T \frac{\partial \beta}{\partial P}\right)+\frac{f \beta^{2}-1}{\beta\left(f-\beta^{2}\right)}+ \\
&\left.+\left(\frac{4 \beta^{3}(1-f) F+\left[f+\left(f^{2}-3\right) \beta^{2}+f \beta^{4}\right] P}{\beta^{2}\left(f-\beta^{2}\right)^{2}}\right) \frac{\partial \beta}{\partial P}+\left(\frac{\left(1+\beta^{2}\right) P-2 \beta F}{\gamma^{2} \beta\left(f-\beta^{2}\right)^{2}}\right) \frac{\partial f}{\partial P}\right], \\
& b=\kappa U_{1}\left[\frac{a_{R} T^{3}}{\beta^{2}}\left(4 \beta \frac{\partial T}{\partial F}-T \frac{\partial \beta}{\partial F}\right)+\frac{2-f-\beta^{2}}{\left(f-\beta^{2}\right)}+\right. \\
&\left.+\left(\frac{4 \beta^{3}(1-f) F+\left[f+\left(f^{2}-3\right) \beta^{2}+f \beta^{4}\right] P}{\beta^{2}\left(f-\beta^{2}\right)^{2}}\right) \frac{\partial \beta}{\partial F}+\left(\frac{\left(1+\beta^{2}\right) P-2 \beta F}{\gamma^{2} \beta\left(f-\beta^{2}\right)^{2}}\right) \frac{\partial f}{\partial F}\right], \\
& c=\kappa U_{1}\left[4 a T^{3} \frac{\partial T}{\partial P}+1+\frac{F}{\beta^{2}} \frac{\partial \beta}{\partial P}\right], \\
& d=\kappa U_{1}\left[4 a T^{3} \frac{\partial T}{\partial F}-\frac{1}{\beta}+\frac{F}{\beta^{2}} \frac{\partial \beta}{\partial F}\right] .
\end{aligned}
\end{gathered}
$$

From algebraic equations (3-4) we can find:

$$
\begin{gathered}
\frac{\partial \beta}{\partial P}=-\frac{(n+1) \beta}{G(\beta, F, P, n)}, \\
\frac{\partial \beta}{\partial F}=\frac{1+n \beta^{2}}{G(\beta, F, P, n)}, \\
\frac{1}{m} \frac{\partial T}{\partial P}=\gamma \beta^{2} \frac{U_{3}-F}{U_{1} G(\beta, F, P, n)},
\end{gathered}
$$




$$
\begin{gathered}
\frac{1}{m} \frac{\partial T}{\partial F}=-\frac{G(\beta, F, P, n)+\beta \gamma^{2}\left(1+n \beta^{2}\right)\left(U_{3}-F\right)}{(n+1) \gamma U_{1} G(\beta, F, P, n)}, \\
G(\beta, F, P, n)=\beta \gamma U_{1}+2 n \beta\left(U_{3}-F\right)-(n+1)\left(U_{2}-P\right) .
\end{gathered}
$$

The sign of the determinant $P=a d-b c$ determines the nature of the singular points $F_{x}, P_{x}$. If $P<0$, we have a saddle point, $P>0$ node $\left((a+d)^{2}>P\right)$ or focus $\left((a+d)^{2}<P\right)$.

Sadle singular points can not be connected by one separatrix (Andronov et al. 1987) and the boundary value problem must be solved in this case to find the hydro and radiation quantity profiles.

For the M1 closure the analysis is similar to the Eddington closure case because the derivatives $\partial f / \partial F, \partial f / \partial P$ for the M1 closure are proportional to radiation flux in the comoving frame $F_{0}$ and in the equilibrium points equal to zero

In the solution of the radiative transfer equation the Eddington factor $f$ is not constant and the singular points behavior is different from the Eddington closure case $(f=1 / 3)$.

Partial derivatives $\partial f / \partial F$ and $\partial f / \partial P$ can be found from

$$
d f=\frac{\partial f}{\partial F} d F+\frac{\partial f}{\partial P} d P
$$

and coupled with

$$
\frac{d F}{d P}=\frac{c}{d-l},
$$

where $l$ is an eigenvalue of the matrix of the linearized system (A18 A19).

The total derivatives $d f / d F$ and $d f / d P$ can be estimated from radiative transfer equation in the limit of small velocities $\beta$. In the case of a gas-dominated flow the solution of the radiative transfer equation can be written as a formal solution (see Appendix B), and for an asymptotic solution, where $d E_{0} / d x=\left|d F_{0} / d x\right|=d P_{0} / d x$ we have

$$
\begin{aligned}
& \frac{d f}{d P}=\frac{d\left(P_{0} / E_{0}\right)}{d P}=\frac{2}{3 E_{0}} \frac{d P_{0}}{d P}, \\
& \frac{d f}{d F}=\frac{d\left(P_{0} / E_{0}\right)}{d F}=\frac{2}{3 E_{0}} \frac{d P_{0}}{d F},
\end{aligned}
$$

Finally, $d F$ and $d P$ can be found by differentiating the Lorentz transformations (9) and the equation for velocity:

$$
\begin{gathered}
d F=\gamma^{4}\left(4 \beta F_{0}+\left(1+\beta^{2}\right)\left(P_{0}+E_{0}\right)\right) d \beta+\gamma^{2}\left(\left(1+\beta^{2}\right) d F_{0}+\beta\left(d P_{0}+d E_{0}\right)\right) \\
\left.d P=4 \gamma^{4}\left(\beta\left(E_{0}+P_{0}\right)+\left(1+\beta^{2}\right) F_{0}\right) d \beta+\gamma^{2}\left(2 \beta d F_{0}+d P_{0}+\beta^{2} d E_{0}\right)\right) \\
d \beta=\frac{\partial \beta}{\partial F} d F+\frac{\partial \beta}{\partial P} d P
\end{gathered}
$$

For the radiation dominated flow $d E_{0} / d P_{0}$ and $d F_{0} / d P_{0}$ can be found by a numerical solution of the radiative transfer equation.

\section{B. FORMAL SOLUTION OF THE EQUATION OF RADIATIVE TRANSFER FOR A STEP FUNCTION SOURCE FUNCTION}

The formal solution of the equation of transfer in a static medium of the layer with a finite optical thickness (see Chandrasekhar (1950)) reduce to

$$
\begin{gathered}
I(\tau,+\mu, \phi)=I\left(\tau_{1}, \mu, \phi\right) \exp ^{-\left(\tau_{1}-\tau\right) / \mu}+\int_{\tau}^{\tau_{1}} S(t, \mu, \phi) \exp -(t-\tau) / \mu \frac{d t}{\mu}, \\
I(\tau,-\mu, \phi)=I(0,-\mu, \phi) \exp ^{-\tau / \mu}+\int_{0}^{\tau} S(t,-\mu, \phi) \exp -(\tau-t) / \mu \frac{d t}{\mu} .
\end{gathered}
$$

where $S(t, \mu, \phi),(1 \geq \mu \geq 0)$ is the source function. If we assume Kirchhoff's law and a step source function

$$
\begin{aligned}
& S=B_{R},(x>0), \\
& S=B_{L},(x<0),
\end{aligned}
$$

then intensities and radiation moments can be found as:

$$
I_{R,-}=B_{R},
$$




$$
\begin{gathered}
I_{R,+}=B_{L} \exp \left(-\tau_{R} / \mu\right)+B_{R} \int_{0}^{\tau_{R}} \exp \left(-\left(\tau_{R}-t\right) / \mu\right) \frac{d t}{\mu} \\
J_{R}=\frac{1}{2} \int_{-1}^{1} I_{R} d \mu=\frac{1}{2} \int_{-1}^{0} I_{R,-} d \mu+\frac{1}{2} \int_{0}^{1} I_{R,+} d \mu= \\
=\frac{1}{2}\left[B_{R}+B_{L} \int_{0}^{1} \exp \left(-\tau_{R} / \mu\right) d \mu+B_{R} \int_{0}^{\tau_{R}} \exp \left(-\left(\tau_{R}-t\right) / \mu\right) \frac{d t}{\mu}\right]=\frac{1}{2}\left[2 B_{R}+\left(B_{L}-B_{R}\right) E_{2}\left(\tau_{R}\right)\right] \\
H_{R}=\frac{1}{2} \int_{-1}^{1} I_{R} \mu d \mu=\frac{1}{2} \int_{-1}^{0} I_{R,-} \mu d \mu+\frac{1}{2} \int_{0}^{1} I_{R,+} \mu d \mu=\frac{1}{2}\left[\left(B_{L}-B_{R}\right) E_{3}\left(\tau_{R}\right)\right] \\
K_{R}=\frac{1}{2} \int_{-1}^{1} I_{R} \mu^{2} d \mu=\frac{1}{2} \int_{-1}^{0} I_{R,-} \mu^{2} d \mu+\frac{1}{2} \int_{0}^{1} I_{R,+} \mu^{2} d \mu=\frac{1}{2}\left[\frac{2}{3} B_{R}+\left(B_{L}-B_{R}\right) E_{4}\left(\tau_{R}\right)\right]
\end{gathered}
$$

The values of the radiation moments at $x<0$ can be found similarly. 